\title{
Performance Evaluation of Snoop Protocol for Wireless Networks
}

\author{
Sherif Moussa, Mohammad Wahba, and Salah Ramadan
}

\begin{abstract}
The performance of TCP decreases when high bit error rate exists over wireless link. TCP responds by mistake to all packet losses by invoking congestion control algorithm, resulting in degraded end-to-end performance. Several solutions have been proposed to improve the performance of transmission control protocol (TCP) over wireless link. In this paper, the implementation of snoop protocol, as a TCP-aware link level over wireless networks, and conventional TCP are compared using wired infrastructure. All simulations were performed using network simulator 2 (NS-2). The simulation results focus on key performance metrics such as throughput, congestion control, end-to-end time delay, and packet loss rate.
\end{abstract} ns2.

Index Terms-TCP, snoop protocol, wireless networks,

\section{INTRODUCTION}

The transmission control protocol (TCP) has grown to be the most important communication protocol which affects the stability of the data networks. However, when TCP works over wireless environments several well known problems arises such as congestion, packet losses due to high Bit Error Rates (BERs), signal fading, and hand-off procedures. Hence, TCP misinterprets those losses, and invoke unnecessarily congestion control algorithms, yielding low throughputs.

Employing TCP over wireless networks has been introduced in [1], [2] in which both end-to-end throughput and radio link utilization decrease due to the mismatch of the dynamic properties of TCP and the wireless links.

Several schemes have been proposed to alleviate the effects of non-congestion-related losses on TCP performance over wireless networks [3]-[8]. These schemes classify the proposed protocols into three categories: link layer protocols, end-to-end protocols and split connection protocols.

The link layer protocols are employed between base station and mobile host where the link related losses from the TCP sender use reliable local retransmission adapted to the wireless links yielding a significant increase in the performance e.g. snoop protocol. In contrast, the end-to-end protocols deal with the TCP sender handle packets losses all the way through till the mobile host. The split connection protocols, as a third type, split each TCP connection

Manuscript received October 11, 2014; revised March 21, 2015.

S. Moussa is with the School of Engineering, Canadian University of Dubai, Dubai, UAE (e-mail: smoussa@cud.ac.ae).

M. Wahba is with The National Authority for Remote Sensing and Space Science, Cairo, Egypt (e-mail: m_w_khalaf@yahoo.com).

S. Ramadan is with the School of Engineering, Azhar University, Cairo, Egypt (e-mail: salah.ramadan@yahoo.com). between the sender and the receiver into two separate connections at the base station. The first connection is the TCP connection between the sender and the base station, and the second connection is the connection between the base station and the receiver. Each connection deals with its lost packets.

There are few studies on improving TCP performance over wireless networks. Balakrishnan et al. [3] defined most of proposed schemes for wireless link problems. The authors in [9] selected the radio link protocol (RLP) as a mechanism, over link level to improve TCP over wireless link where the effects of RLP on TCP are studied. Majid et al. [10] studied TCP over wireless networks, by adapting the transmission rate according to the receiver response or the receiver feedback. Lucas et al. [11] Implemented the cross layer design (CLD) algorithm over Wi-Max network, and used snoop agent to increase TCP throughput with decease time out.

In this paper, a new modified routing code function in NS-2 is added, it monitor all packets passes through Base Station for each connection, and then the NS-2 simulator [12] is used to compare between both snoop and regular TCP protocols.

The impact of TCP parameters such as average End-toEnd delay, throughput and packet loss ratio are observed and evaluated. Simulation model is used to verify that the wireless networks can use the snoop protocol to overcome the wireless link errors through link layer retransmissions. Thus a decrease in the delay is observed. In addition, studying TCP and its problems over wireless networks is introduced.

The rest of this paper is organized as follows. Section II presents a brief description of the snoop protocol. The network model and the implementation of snoop protocol at the base station are introduced in Section III. Section IV presents the simulation details and the simulation results. Section V concludes this paper.

\section{SNOOP TCP PROTOCOL}

The Snoop protocol [3] runs on a Snoop agent that is implemented in a base station or a wireless device. The agent monitors packets that pass through the base station and caches them. After caching, the agent forwards packets to their packets destinations and monitors the corresponding acknowledgements. The role of the Snoop agent is to cache data packets for a TCP connection, when data packets are lost (indicated by the reception of duplicate ACKs), the Snoop agent retransmits those packets. It retransmits them locally without forwarding the Duplicate ACKs to the sender. Hence, since the TCP layer is not aware of the 
packet loss, the congestion control algorithm is not triggered. In addition, the Snoop agent starts a retransmission timer [13], [14] for each TCP connection. When the retransmission timer expires, the agent retransmits the packets that have not been acknowledged yet. This timer is called a persist timer because, unlike TCP retransmission timer, it has a fixed value.

A Snoop agent implementation in a base station is shown in Fig. 1. This network consists of two parts, the first network is wired network (fixed host - router), and the second network is wireless network (base station with snoop agent - mobile nodes) in which a Snoop agent is implemented in a base station.

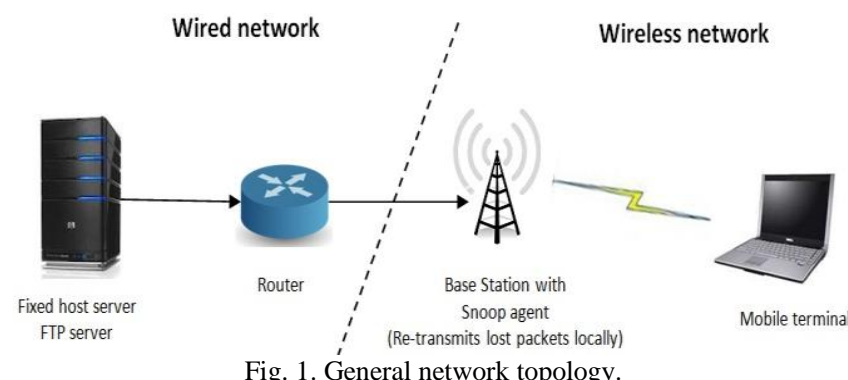

The flow chart of snoop packet arrival from upper TCP/IP layer is shown in Fig. 2.

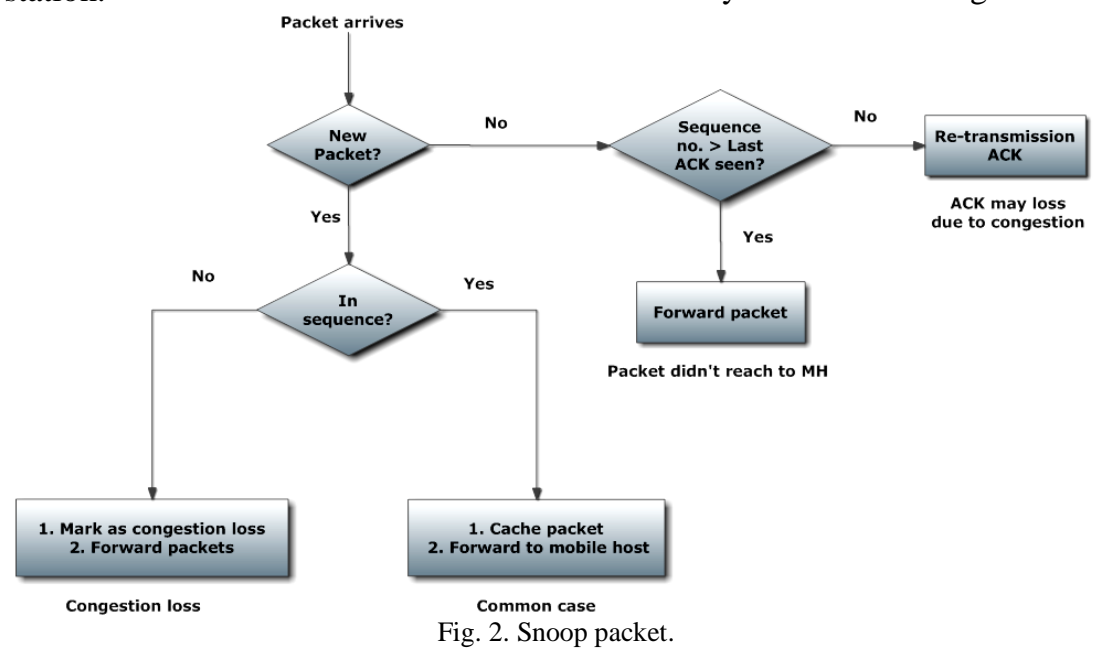

Snoop module processes packets arrived from $\mathrm{FH}$, that TCP implements a sliding window scheme based on congestion window. TCP sends byte by byte, and each byte of data has an associated sequence number.

When a new packet in the normal increasing in sequence arrives at base station. In this case packet is added to snoop cache and forwarded to the mobile host (common case).

In case of the packet arrived is out-of-sequence, that is lost earlier in the wired network due to congestion or was delivered out-of-order by the wired network, subsequently.

1) The packet loss is marked as congestion (sender retransmission).

2) Forward to mobile host.

When arrived packet isn't new packet and sequence number of the incoming packet $>$ the last packet ACKed by mobile host: it is very likely that this packet didn't reach the mobile host earlier, and as a result it will be forwarded.

Sequence number of incoming packet $<$ sequence number of the last ACKed packet by mobile host: this packet has already been received by the mobile host, because ACK must have been lost in the wired part of the network. Hence base station generates and sends an ACK to fixed host.

Fig. 3 shows, when a packet arrives from the MAC layer to the Snoop agent, the process transits from the Waitstate to the Snoop ACK state. The packet is processed depending on the acknowledgment sequence number:

When the incoming acknowledgment has a sequence number isn't duplicating DupACK number, it's higher than the last received. Subsequently:

1) Free appropriate buffer in cache.

2) Forward to fixed host.

3) Update round trip time (RTT) estimates.
Check if the DupACK isn't the first one coming. This is an acknowledgment with sequence number lower than the last received. This rarely happens and the acknowledgment is discarded.

If the first one coming, it is an acknowledgment that is identical to the last received. This causes the Snoop agent to assume that packets that have been sent with higher sequence number were lost. The Snoop agent will retransmit all packets starting with the first lost packet.

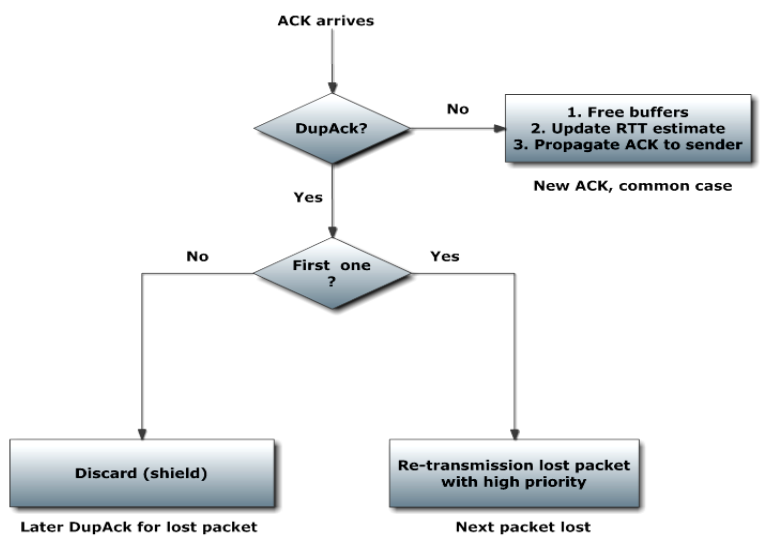

Fig. 3. Snoop ACK.

\section{Simulation MODEL}

This paper discusses the wireless LAN environment that is typical to cellular wireless environment having the same impacts on TCP performance. NS-2 simulator visualization of the network model is shown in Fig. 4.

The small numbered circle represents mobile nodes, 
while the other unnumbered small circle represents base station, fixed host and LAN.

The simulated network shown in Fig. 5 consists of:

1) Domain: number of domains in this topology is two:
- One for the wired nodes.

- One for the wireless.

2) Cluster: number of clusters in each of these domains is defined as "2 1".

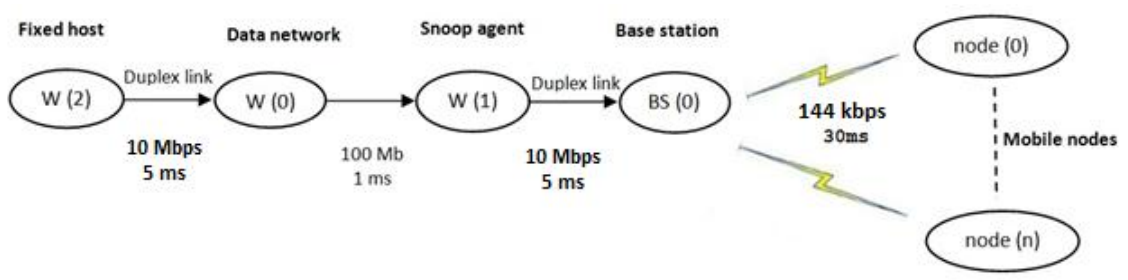

Fig. 4. The network topology.

" 2 " indicates the first domain has two clusters for wired, these are:

1) Cluster 1 contains: $W(2), W(0)$ and $W(1)$.

2) Cluster 2 contains: $B S(0)$ as a gateway between wireless and wired domains, and allow packets to be exchanged between the two types of network.

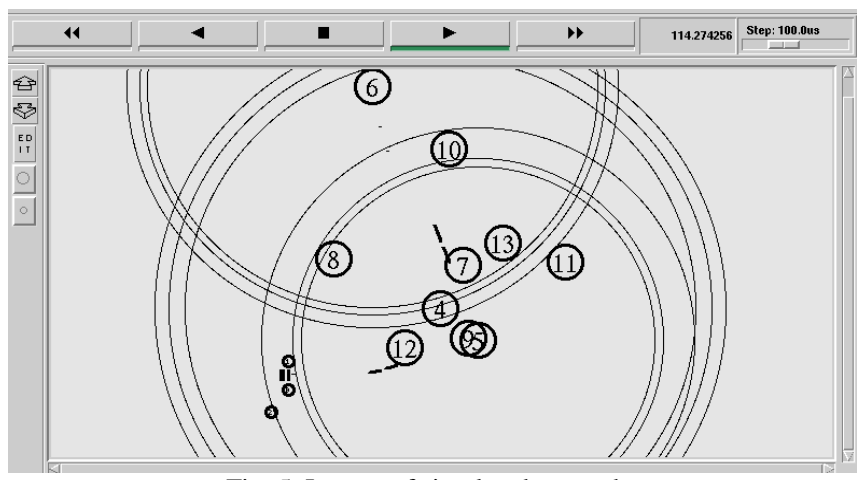

Fig. 5. Layout of simulated network.

The second domain has one cluster for wireless.

Cluster 1 contains: all nodes from node $(0)$ to node $(n)$.

Table I shows the simulation parameters. The simulation model assumes that the Snoop agent is implemented on node $W(1)$ as shown in Fig. 4.

TABLE I: SIMULATION PARAMETERS

\begin{tabular}{ll}
\hline \hline Simulation parameter & Value \\
\hline Application & FTP \\
Simulation area & $50 \times 50,100 \times 100,200 \times 200,300 \times 300$, \\
& $350 \times 350,400 \times 400$ \\
Time to stop simulation & $50,100,150,200,250,300,350$ \\
Number of domain & $3,7,10,15,20,25,30$ \\
Number of clusters & 111 \\
Number of nodes/cluster & $3,7,10,15,20,25,30$ \\
& $W(0) \quad 0.0 .1$ \\
Address hierarchal address & $W(1) \quad 0.0 .2$ \\
& $W(2) 2.0 .0$ \\
Error rate & $B S(0) 1.0 .0$ \\
Interface queue & $3 \%$ \\
Max. packet in interface queue & 100 \\
\hline \hline
\end{tabular}

\section{Simulation Results}

The results were extracted from trace files generated by NS-2, based on the model described above. $\mathrm{C}++, \mathrm{TCl}$ and OTcl scripts were used for this purpose. Many simulation scenarios are carried out to compare between snoop and conventional TCP protocols.
The impact of changing the number of nodes on the throughput is shown in Fig. 6, the minimum throughput occurred at 3 wireless nodes at which throughput of snoop protocol increases by 13000 bps, while the maximum throughput occurred at 30 wireless nodes at which the throughput of snoop protocol exceeds the conventional TCP by 3784.88 bps.

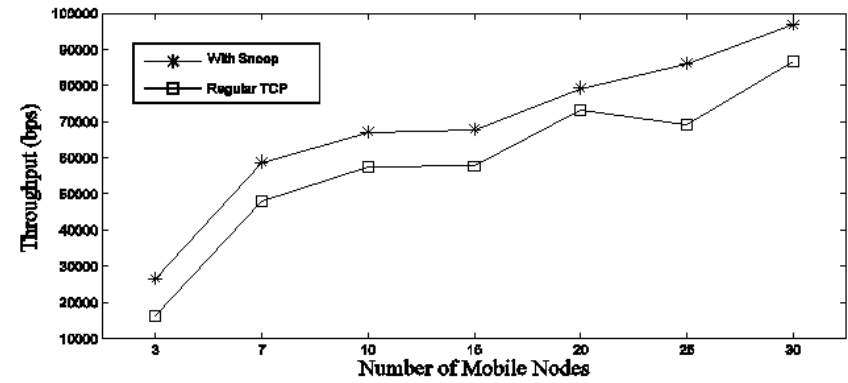

Fig. 6. Throughput versus number of mobile nodes.

Fig. 7 shows the number of nodes against the packet loss, at 30 wireless nodes the packet loss ratio of conventional TCP is $65 \%$, while it is $23 \%$ for snoop protocol, this is about $42 \%$ reduction of packet loss.

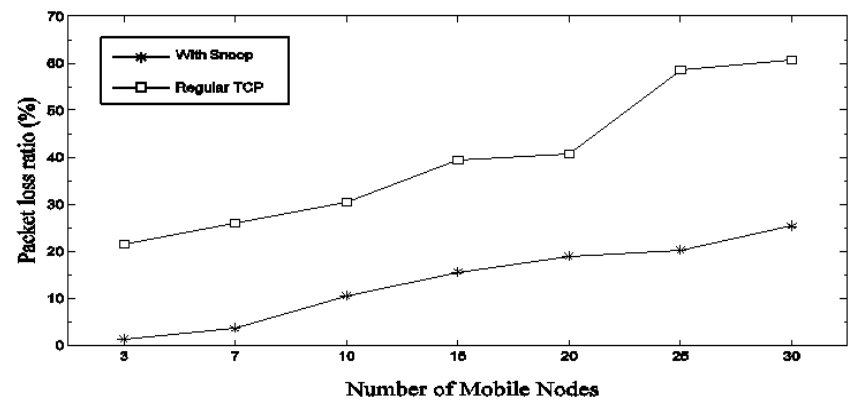

Fig. 7. Packet loss versus number of mobile nodes.

Fig. 8 shows the average end-to-end delay as a function of number of mobile nodes. Snoop protocol achieves less average delay for the same number of nodes.

This is due to the processing, queuing and link delay are done between base station and mobile station without the need to go back to the TCP sender, unlike conventional $\mathrm{TCP}$, which is end to end delay calculation.

Finally, Fig. 9 shows the congestion window over time, at simulation time of 20 seconds packet losses suddenly occurs; consequently conventional TCP is considering this loss due to congestion in the network and decreases its congestion window. This consideration is valid for wired network, however, it is not true for wireless network where 
packet loss could be caused by bit errors rate from environment effect (fading, multipath or delay). The snoop protocol as shown in the figure is able to overcome this false indication in the wireless network; it can be observed that a consistent congestion window is maintained when snoop protocol is used.

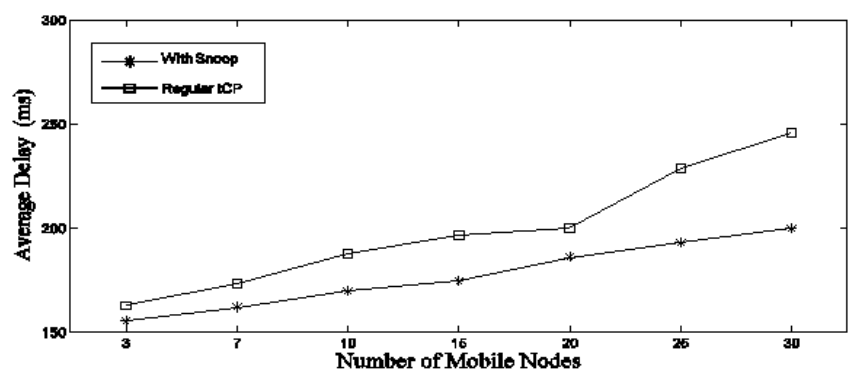

Fig. 8. Average delay versus number of mobile nodes.

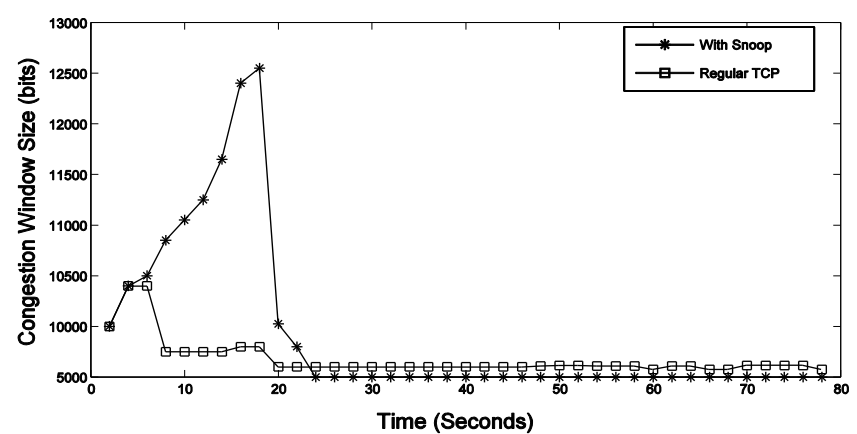

Fig. 9. Congestion window versus time.

\section{CONCLUSION}

In this paper, snoop protocol, as a TCP-aware link level over wireless networks, and conventional TCP are compared using NS-2 simulation model. The simulation model incorporated various parameters like throughput, congestion window, delay time and sequence trace for both protocols. It is observed that snoop protocol greatly enhances the performance of TCP over wireless networks. Additionally the snoop agent is able to shield the sender from decreasing its congestion window by misinterpreting wireless error losses as congestion. Finally, it is found that the snoop protocol is most effective over moderate error rates.

\section{REFERENCES}

[1] 3GPP2 home page. [Online]. Available: http://www.3GPP2.org

[2] E. Yanmaz, S. C. Wei, and O. K. Tonguz, "Performance enhancement techniques for TCP over wireless links," in Proc. 2005 IEEE 61st Vehicular Technology Conference, 2005, pp. 2245-2249.

[3] H. Balakrishnan, V. N. Padmanabhan, S. Seshan, and R. H. Katq, "A comparison of mechanisms for improving TCP Performance over. wireless links," Transactions on Networking, vol. 5, no. 6, pp. 756769, Dec 1997.

[4] T. Goff, J. Moronski, D. S. Phatak, and V. Gupta, "Freeze-TCP: A true end-to-end TCP enhancement mechanism for mobile environments," in Proc. the IEEE INFOCOM, Mar. 2000, pp. 1537 1545 .

[5] K. Brown and S. Singh, "M-TCP: TCP for mobile cellular networks," Department of Computer Science, University of South Carolina Columbia, July 29, 1997.
[6] B. R. Badrinath and A. Bakre, "I-TCP: Indirect TCP for mobile hosts," in Proc. the 15th Int'l Conference on Distributed Computing, May 1995, pp. 136-143.

[7] S. Vagala and M. Vehicular, "The TCP SACK-aware snoop protocol for TCP over wireless networks," in Proc. the Vehicular Technology Conference, October 2003, pp. 2624-2628.

[8] A. Kumar, "Comparative performance analysis of versions of TCP in a local network with a Lossy link," IEEE Trans. Networking, vol. 6 , no. 4, pp. 485-498, Aug. 1998.

[9] H. T. Lin and S. K. Das, "Performance study of link layer and MAC layer protocols to support TCP in 3G CDMA systems," IEEE Transactions on Mobile Computing, vol. 4, no. 5, September/October 2005.

[10] M. Ghaderi, D. Towsley, R. Cruz, A. Sridharanand, and H. Zang, "TCP A-ware resource allocation in CDMA networks," in Proc. MobiCom 06, 2006, Los Angeles, California, USA.

[11] L. D. P. Mendes and J. M. C. Brito, "Effects of the use of an IEEE 802.11 snoop agent on TCP timeouts," in Proc. 2010 IEEE International Conference on Wireless Communications, Networking and Information Security, 2010, pp. 493-497.

[12] T. Issariyakul and E. Hossain, Introduction to Network Simulator NS2, Springer Science Business Media, 2009.

[13] V. Zhang and G. Feng, "A new method to improve the TCP performance in wireless cellular networks," National Key Laboratory of Communication, University of Electronic Science and Technology of China, 2009.

[14] Y.-B. Cho, G.-S. Won, and S.-J. Cho, Improvement of TCP Throughput with the Snoop + A Protocol, 2006.

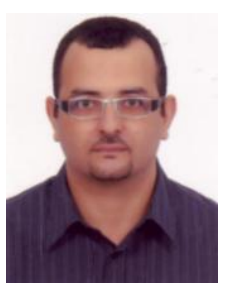

Sherif Moussa received his Ph.D. degree in electrical and computer engineering from University of Quebic Trois-Riviers, Canada, and his M.Sc. degree in electrical and computer engineering form University of Waterloo, Canada. His research areas are wireless communication, computer networks, and VLSI design. His research specifically focuses on MIMOOFDM algorithms, multiple access OFDM, FPGA design and optimization. He is currently an assistan professor at School of Engineering, Canadian University of Dubai, UAE Prior to joining CUD, he was a lecturer at School of Engineering, Centennial College, Toronto, Canada. Dr. Sherif is a cisco academy instructor for CCNA and a SUN certified programmer for Java 2 Platform.

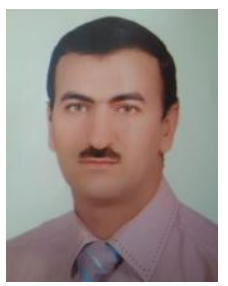

Mohammad Wahba Khalaf was born at Monofia, Egypt in 1980. He received his first degree in systems and computer engineering from Faculty of Engineering, Al Azhar University, Cairo, Egypt in 2005 and the master degree in 2013 from Systems and Computer Dept., Al Azhar University. Currently he is pursuing his $\mathrm{PhD}$ degree in signal processing of radar remote sensing for buried target detection at Al-Azhar University. Mr. Khalaf currently is a telemetry senior engineer for remote sensing satellite.

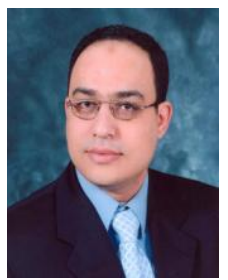

Salah M. Ramadan received his M.S. and Ph.D degrees in systems and computers engineering from Al-Azhar University in 2002 and 2005, respectively. Since 2012, he is an associate professor in systems and computers engineering at Al-Azhar University. Dr. Ramadan completed his post-doctoral research in 2007 and 2008 at the Computer Science and Engineering Department, School of Engineering, the Southern Methodist University at Dallas, TX, USA. His research interests include mobile computing, cellular networks, senso networks, cognitive radio networks, and internet services and applications. 